Appears in Phys. Rev. Lett.

\title{
DIRECT HOPF BIFURCATION IN PARAMETRIC RESONANCE OF HYBRIDIZED WAVES
}

\author{
Franz-Josef Elmer \\ Institut für Physik, Universität Basel, CH-4056 Basel, Switzerland
}

(January 29, 1996)

\begin{abstract}
We study parametric resonance of interacting waves having the same wave vector and frequency. In addition to the well-known period-doubling instability we show that under certain conditions the instability is caused by a Hopf bifurcation leading to quasiperiodic traveling waves. It occurs, for example, if the group velocities of both waves have different signs and the damping is weak. The dynamics above the threshold is briefly discussed. Examples concerning ferromagnetic spin waves and surface waves of ferro fluids are discussed.
\end{abstract}

PACS numbers: 03.40.Kf, 76.50.+g, 47.20.-k

Typeset using REVTEX 
Parametric resonance is an instability phenomenon that is responsible for the excitation of modes of a weakly damped system due to periodic modulation of parameters which determine the frequencies of the modes [1]. The prototypical example is the vertically driven pendulum where the modulated parameter is the acceleration of gravity [2]. In spatially extended systems these modes are waves. Experimentally parametric resonance is well-known for spin waves (Suhl instabilities) [3 5] and surface waves of fluids (Faraday instability) [6 8].

Let us briefly summarize the well-known behavior of the one-wave case where only one wave type is involved. The ground state (e.g., the flat surface of a fluid) becomes unstable if the driving (i.e., modulation) amplitude exceeds some threshold which is proportional to the damping constant. The instability is caused by the excitation of waves fulfilling the first-order parametric resonance condition

$$
\Omega(\mathbf{k})=\frac{\omega}{2},
$$

where $\Omega(\mathbf{k})$ is the frequency of the wave with wave number $\mathbf{k}$ (i.e., the dispersion relation) and $\omega$ is the modulation frequency. At the instability threshold a period-doubling bifurcation occurs because the wave solution has a period which is two times the period of the modulation. That is, in the stroboscopic map (i.e., the map which gives the state at time $t=2(m+1) \pi / \omega$ provided the state at $t=2 m \pi / \omega$ is known $)$ a period-two orbit bifurcates.

How does this one-wave picture change if two types of waves with different dispersion relations $\Omega_{1}$ and $\Omega_{2}$ are involved? There is in principle no change as long as the manifolds defined by $\Omega_{j}(\mathbf{k})=\omega / 2$ do not intersect. At such intersection lines two different waves with the same wave vector fulfill the parametric resonance condition. Interaction leads to the well-known effect of hybridization where the pure waves are replaced by mixtures of them. Although hybridization avoids the intersection of the above-mentioned manifolds they still come very close together with a minimum distance proportional to the strength of interaction. Below we will see that under certain very general conditions a direct Hopf bifurcation occurs. It leads to quasiperiodic motion right at the onset of parametric resonance. 
The wave lengths of waves which are excited due to parametric resonance are usually large compared to the atomic scales. Their dynamics are described by macroscopic field equations like the Navier-Stokes equations for fluids or the Landau-Lifshitz equations for magnets. Formally the fields can be put together into a single vector field $\mathbf{u}(\mathbf{r}, t)$, called order parameter. In the limit of weak damping universal equations of motion so-called amplitude equations can be derived [1]. They describe the spatio-temporal evolution of the amplitudes of waves fulfilling the parametric resonance condition. The amplitude equations are derived from a multiple-scale perturbation theory where the damping constant is the smallness parameter. For the field $\mathbf{u}$ we make the ansatz:

$$
\begin{aligned}
\mathbf{u}(\mathbf{r}, t)= & \left(A_{+} \mathbf{u}_{+}+A_{-} \mathbf{u}_{-}\right) e^{i(k x-\omega t / 2)}+ \\
& +\left(B_{+} \mathbf{v}_{+}+B_{-} \mathbf{v}_{-}\right) e^{i(k x+\omega t / 2)}+\text { c.c. },
\end{aligned}
$$

where $A_{ \pm}$and $B_{ \pm}$are the slowly varying amplitudes of the right and left waves, respectively. The corresponding wave solutions are $\mathbf{u}_{ \pm}$and $\mathbf{v}_{ \pm}$. In general they depend on $y$ and $z$. The index \pm corresponds to the different types of waves. The cross point of their dispersion relations $\Omega_{ \pm}(k)$ are denoted by $k_{0}$ and $\omega_{0}$, i.e., $\Omega_{ \pm}\left(k_{0}\right)=\omega_{0}$. It is assumed that the modulation amplitude $h$, the detuning $\omega / 2-\Omega(k)$, the amplitudes of the waves, and the coupling strength between different types of waves are of first order in the damping constant. In the one-wave case the amplitude equations for $A_{ \pm}$and $B_{ \pm}$are well-known [1,9.10]. Coupling between + and - waves lead to additional linear cross terms. Because of the hamiltonian nature of these coupling $\mathbf{u}_{ \pm}$and $\mathbf{v}_{ \pm}$can always be chosen in such a way that the linear part of the amplitude equations reads:

$$
\begin{aligned}
& \dot{A}_{ \pm}=-\Gamma_{ \pm} A_{ \pm}+i h \alpha_{ \pm} B_{ \pm}+i \epsilon e^{ \pm i \chi} A_{\mp}, \\
& \dot{B}_{ \pm}=-\Gamma_{ \pm}^{*} B_{ \pm}-i h \alpha_{ \pm} A_{ \pm}+i \epsilon e^{\mp i \chi} B_{\mp},
\end{aligned}
$$

with

$$
\Gamma_{ \pm}=\gamma_{ \pm}+i\left(\Omega_{ \pm}-\omega / 2\right), \quad \gamma_{ \pm}>0
$$


where $\gamma_{ \pm}, \Omega_{ \pm}$, and $\alpha_{ \pm}$are real numbers denoting for each wave type the damping constant, the dispersion relation, and the strength of modulation, respectively, $\epsilon$ and $\chi$ are the strength and the phase of the coupling, respectively. These parameters are in general functions of the wave number $k$. The amplitude and frequency of modulation are denoted by $h$ and $\omega$, respectively.

The ground state $A_{ \pm}=B_{ \pm}=0$ becomes unstable if a solution $A_{ \pm}=a_{ \pm} \exp (\lambda t)$, $B_{ \pm}=b_{ \pm} \exp (\lambda t)$ exists with $\operatorname{Re} \lambda>0$. For increasing $h, \lambda$ will eventually cross the imaginary axis defining a bifurcation point. Since the coefficients of (3) depend on $k$ these points become functions of $k$ called neutral curves. There are two different types of neutral curves possible: (i) Neutral curves $h_{S}(k)$ where $\lambda=0$ (soft-mode or stationary instability) and (ii) neutral curves $h_{H}(k)$ where $\operatorname{Im} \lambda \equiv \omega_{H} \neq 0$ (hard-mode or oscillatory instability). In the stroboscopic map they correspond to period-doubling bifurcation and Hopf bifurcation, respectively. The neutral curves $h_{S}$ and $h_{H}$ are the real solutions of

$$
E h^{4}-F h^{2}+G=0
$$

with $E, F$, and $G$ given by

$$
\begin{aligned}
E_{S} & =\left(\alpha_{+} \alpha_{-}\right)^{2}, \\
F_{S} & =\left|\alpha_{+} \Gamma_{-}\right|^{2}+\left|\alpha_{-} \Gamma_{+}\right|^{2}-2 \alpha_{+} \alpha_{-} \epsilon^{2} \cos 2 \chi, \\
G_{S} & =\left|\Gamma_{+} \Gamma_{-}+\epsilon^{2}\right|^{2},
\end{aligned}
$$

and

$$
\begin{aligned}
E_{H}= & \gamma_{+} \gamma_{-}\left(\alpha_{+}^{2}-\alpha_{-}^{2}\right)^{2}, \\
F_{H}= & 2 \gamma_{+} \gamma_{-}\left[\left(\alpha_{+}^{2}-\alpha_{-}^{2}\right)\left(\left|\Gamma_{+}\right|^{2}-\left|\Gamma_{-}\right|^{2}\right)+\right. \\
& \left.+2\left(\gamma_{+}+\gamma_{-}\right)\left(\gamma_{-} \alpha_{+}^{2}+\gamma_{+} \alpha_{-}^{2}\right)\right]+ \\
& +\left(\gamma_{+}+\gamma_{-}\right)^{2}\left(\alpha_{+}^{2}+\alpha_{-}^{2}+2 \alpha_{+} \alpha_{-} \cos 2 \chi\right) \epsilon^{2}, \\
G_{H}= & \gamma_{+} \gamma_{-}\left[\left(\left|\Gamma_{+}\right|^{2}-\left|\Gamma_{-}\right|^{2}\right)^{2}+\right. \\
& \left.+4\left(\gamma_{+}+\gamma_{-}\right)\left(\gamma_{-}\left|\Gamma_{+}\right|^{2}+\gamma_{+}\left|\Gamma_{-}\right|^{2}\right)\right]
\end{aligned}
$$




$$
+\left|\left(\gamma_{+}+\gamma_{-}\right)\left(\Gamma_{+}+\Gamma_{-}\right) \epsilon\right|^{2},
$$

respectively. The solution $h_{H}$ is relevant only if the Hopf frequency $\omega_{H}$ given by

$$
\omega_{H}^{2}=\epsilon^{2}+\frac{\gamma_{-}\left|\Gamma_{+}\right|^{2}+\gamma_{+}\left|\Gamma_{-}\right|^{2}-\left(\gamma_{-} \alpha_{+}^{2}+\gamma_{+} \alpha_{-}^{2}\right) h_{H}^{2}}{\gamma_{+}+\gamma_{-}}
$$

is real.

In the noninteracting case (i.e., $\epsilon=0$ ) the characteristic polynomial (5) factorizes into two second-order polynomials and we are back to the one-wave case. The instability is always caused by a real $\lambda$ crossing the imaginary axes and consequently $h_{H}$ does not exist. The neutral curve reads $h_{S}=\left|\Gamma_{ \pm}\right| / \alpha_{ \pm}$. The instability threshold $h_{c}$ (i.e., the absolute minimum) is given by $h_{c}=h_{ \pm} \equiv \gamma_{ \pm} / \alpha_{ \pm}$.

The two-wave case (i.e., $\epsilon \neq 0$ ) is qualitatively much richer. Outside the hybridization area the one-wave picture is still valid. We assume that the threshold is given by the + wave, i.e., $h_{c}=h_{+}$. How does the type and the threshold of the instability change if we sweep through a hybridization area? First it can be rigorously proved that the threshold is always higher than $h_{+}$. This can be easily understood because the coupling of a parametrically excited wave with another wave opens an additional dissipation channel. Thus the driving amplitude has to be increased in order to excite a wave parametrically. In the hybridization region where the coupling is strongest this effect is most pronounced. In the limit of strong damping or weak coupling this effect is of the order $\epsilon^{2}$. In this limit direct Hopf bifurcations are not possible.

For the other limit (i.e., strong coupling or weak damping) we have to distinguish between two cases depending on whether the slopes of the dispersion relations (i.e., the group velocities $\left.c_{ \pm} \equiv d \Omega_{ \pm} / d k\right)$ have the same sign or not. When both signs are identical the neutral curve $h_{S}$ has always two relative minima located near the parametric resonance condition. The reason for that is that the hybridized dispersion relations are monotonic functions of the wave number. Sweeping $\omega$ through the hybridization region one minimum increases whereas the other one decreases [see Fig. [(a)]. There will be a point in the middle near $\omega=2 \omega_{0}$ where both minima are of equal height. This is a co-dimension two bifurcation point where 
a competition between two period-doubling bifurcations for two different values of $k$ takes place. Assuming no $k$-dependence of the group velocities $c_{ \pm}$and the parameters $\gamma_{ \pm}, \alpha_{ \pm}, \epsilon$, and $\chi$ we get approximatively for the threshold in the co-dimension two point

$$
h_{S c}\left(2 \omega_{0}\right)=\frac{\left|c_{-} \gamma_{+}+c_{+} \gamma_{-}\right|}{\sqrt{c_{-}^{2} \alpha_{+}^{2}+c_{+}^{2} \alpha_{-}^{2}-2 c_{+} c_{-} \alpha_{+} \alpha_{-} \cos 2 \chi}} .
$$

The error is of quadratic order in the damping constants. Note that the threshold enhancement $h_{S c}\left(2 \omega_{0}\right)-h_{+}$can be of the same order as $h_{+}$[see Fig. 11(a)].

If the group velocities have different signs the hybridized dispersion relations are no longer monotonic. Thus an interval around $\omega=2 \omega_{0}$ exists where the parametric resonance condition can not be fulfilled. Therefore the threshold $h_{S}$ is much larger than in the case of equal signs (see Fig. 1) because it can not be of the order of the damping constants like (9).

In the limit of strong coupling or weak damping the instability due to a period-doubling bifurcation can be beaten by an oscillatory instability (see Fig. 1). Its neutral curve $h_{H}$ is very well approximated by

$$
h_{H}=\frac{\left|\Gamma_{+}+\Gamma_{-}\right|}{\sqrt{\alpha_{+}^{2}+\alpha_{-}^{2}+2 \alpha_{+} \alpha_{-} \cos 2 \chi}} .
$$

The Hopf frequency $\omega_{H}$ is in leading order given by $\epsilon$ which is half of the hybridization gap at $k=k_{0}$. The threshold

$$
h_{H c}(\omega)=\frac{\gamma_{+}+\gamma_{-}}{\sqrt{\alpha_{+}^{2}+\alpha_{-}^{2}+2 \alpha_{+} \alpha_{-} \cos 2 \chi}}
$$

occurs at

$$
\Omega_{+}\left(k_{H c}\right)+\Omega_{-}\left(k_{H c}\right)=\omega .
$$

Eq. (11) is a good approximation only near $\omega=2 \omega_{0}$. Going away from this point $h_{H c}(\omega)$ increases [see Fig. 1(b)]. It eventually disappears because $h_{H}$ does not exists far away from the hybridization area.

What are the conditions for $h_{H c}<h_{S c}$ ? Because of too many parameters we can not give a complete answer. We will consider only two important case where an easy answer 
is possible. In the first case the group velocities $c_{+}$and $c_{-}$have different signs and $h_{H c}$ is almost always less than $h_{S c}$ because $h_{H c} \sim \mathcal{O}(\gamma)$ and $h_{S c} \sim \mathcal{O}\left(\gamma^{0}\right)$. For equal signs we get an easy conditions if only one wave is driven parametrically (i.e., $\alpha_{-}=0$ ). Here (9) and (11) simplify to $h_{S c}=\left(\gamma_{+}+\gamma_{-}\left|c_{+} / c_{-}\right|\right) / \alpha_{+}$and $h_{H c}=\left(\gamma_{+}+\gamma_{-}\right) / \alpha_{+}$, respectively. Thus the Hopf bifurcation occurs first if $\left|c_{-}\right|<\left|c_{+}\right|$.

In a preliminary study we have also investigated the bifurcating solutions by including usual third-order terms in the amplitude equations [1, [9,10]. The bifurcation at $h_{H}$ is either supercritical or weakly subcritical. The bifurcating solution is similar to the drift solution which occurs in a secondary instability in the one-wave case [1.94. For larger values of $h$ this solution becomes oscillatory unstable. The corresponding Hopf bifurcation leading to a torus solution is either supercritical or weakly subcritical. Note that for the order parameter $\mathbf{u}$ this solution contains three different frequencies. Further increase of $h$ leads via a period-doubling sequence to chaos.

In which physical systems can we expect direct Hopf bifurcation due to parametric resonance? First we distinguish two broad classes of physical systems where hybridization of waves on macroscopic scales takes place. The first class is characterized by at least two different types of waves caused by different physical mechanisms, e.g., spin waves and sound waves in ferromagnets. The phonon-magnon system has already been studied experimentally in ferromagnets by parallel-pumping [11]. Parallel pumping means an additional high-frequency magnetic field parallel to the static one. In parallel-pumping only the spin waves are driven. Unfortunately the usual period-doubling bifurcation comes first because the sound velocity is larger than the magnon group velocity. But the threshold enhancement has been observed [11]. We predict a direct Hopf bifurcation if only sound waves are excited parametrically.

The second class of systems with hybridization is obtained by squeezing a wave with an anisotropic and/or nonmonotonic dispersion relation into a geometry with small transverse extension. Examples are ferromagnetic films in saturating magnetic fields and ferromagnetic fluids in narrow channels with a magnetic field perpendicular to the surface. The 
corresponding dispersion relations are anisotropic [4] and nonmonotonic [12], respectively. The different types of waves in such systems are caused by a discrete set of the transverse component $k_{j}^{\perp}$ of the wave vector. For a wave with a lateral component $k$ the dispersion relation $\Omega_{j}$ is the bulk one where $k$ is replaced by $\sqrt{k^{2}+k_{j}^{\perp 2}}$. Crossings between $\Omega_{j+1}$ and $\Omega_{j}$ occur when $\left|k_{j+1}^{\perp}-k_{j}^{\perp}\right|$ is not too large, i.e., the extension of the system in the transverse direction is not too narrow. The strength of interaction is strongly influenced by the kind of boundary conditions.

We have calculated the threshold for a parallel-pumped, ferromagnetic, insulating film in a uniform magnetic field oriented perpendicular to the film plane [14]. Fig. 22 shows an excellent agreement between a fully numerical calculation and an analytical one within the framework of the amplitude equations (3). The coefficients of the linear terms of the amplitude equations have been already calculated in the literature [15, 16]. On the left hand side of Fig. 2 we see the neutral curves in the hybridization area of the modes $j=0$ and $j=2$. The Hopf bifurcation has clearly the lowest threshold. But unfortunately a non-interacting wave (here the mode $j=1$ on the right hand side) has a slightly lower threshold. Very recently Kostylev et al. [17] have shown in a fully numerical calculation that in tangentially magnetized films the Hopf bifurcation can have the overall lowest threshold.

A promising physical system for experimental verification of the direct Hopf bifurcation is the Faraday instability of a ferrofluid in a static field perpendicular to the surface in order to get a nonmonotonic dispersion relation. The fluid should be in an annular container with a width which is roughly as large as, or slightly larger, than the wavelength of the surface waves at the local maximum of the dispersion relation. At the cross points of dispersion relations of waves with different transverse wave numbers the group velocities have often different signs. Thus the Hopf bifurcation appears first if the damping is not to strong. Without any detailed calculation we can decided whether a non-interacting wave has still a lower threshold or not.

In this letter the influence of hybridization on the parametric resonance of waves has been studied. We have shown that under very general conditions the usual period-doubling 
bifurcation is replaced by a direct Hopf bifurcation leading to quasi-periodic traveling waves. The Hopf frequency is roughly given by half of the hybridization gap.

\section{ACKNOWLEDGMENTS}

I gratefully acknowledge P. Talkner for his critical reading of the manuscript. This work was supported by the Swiss National Science Foundation. 


\section{REFERENCES}

[1] M. C. Cross and P. C. Hohenberg, Rev. Mod. Phys. 65, 851 (1993).

[2] L. D. Landau and E. M. Lifshitz, Mechanics, (Pergamon Press, 1960).

[3] H. Suhl, J. Phys. Chem. Solids 1, 209 (1957).

[4] R. W. Damon, in Magnetism Vol. I, edited by G. T. Rado and H. Suhl (Academics Press, New York, 1963), p. 551.

[5] V. S. L'vov, Wave Turbulence Under Parametric Excitation, (Springer, Berlin, 1994).

[6] M. Faraday, Phil. Trans. R. Soc. London 52, 319 (1831).

[7] J. W. Miles, J. Fluid Mech. 148, 451 (1984).

[8] S. T. Milner, J. Fluid Mech. 225, 81 (1991).

[9] S. Douady, S. Fauve, and O. Thual, Europhys. Lett. 10, 309 (1989).

[10] H. Riecke, Europhys. Lett. 11, 213 (1990).

[11] E. H. Turner, Phys. Rev. Lett. 5, 100 (1960).

[12] V. G. Bashtovoi and R. E. Rosensweig, J. Magn. Magn. Mater. 122, 234 (1993).

[13] F. J. Elmer, appears in Phys. Rev. B. 53, (1996).

[14] In the calculation only the exchange interaction and the dipolar interaction of the spins are included. Furthermore unpinned spins at the sample boundary are assumed [13].

[15] O. G. Bendik, B. A. Kalinikos, and D. N. Chartorizhskii, Fiz. Tverd. Tela 16, 2757 (1974) [Sov. Phys. Solid State 16, 1785 (1975)].

[16] B. A. Kalinikos and A. N. Slavin, J. Phys. C 19, 7013 (1986).

[17] M. P. Kostylev, B. A. Kalinikos, and H. Dötsch, J. Magn. Magn. Mater. 145, 93 (1995). 


\section{FIGURES}

FIG. 1. Thresholds and critical wave numbers as functions of the modulation frequency near a hybridization region. The parameters are $\epsilon=0.06, \chi=1.5, \alpha_{+}=4, \alpha_{-}=0.5, \gamma_{+}=0.03$, $\gamma_{-}=0.02, \Omega_{-}=\omega_{0}+0.2\left(k-k_{0}\right)$, and (a) $\Omega_{+}=\omega_{0}+0.7\left(k-k_{0}\right)$, (b) $\Omega_{+}=\omega_{0}-0.7\left(k-k_{0}\right)$. Solid (dashed) lines indicate relative minima of $h_{S}\left(h_{H}\right)$. The absolute minimum is denoted by thick lines.

FIG. 2. The neutral curves for a parallel-pumped insulating ferromagnetic film of thickness $d$. The magnetic field is uniform and perpendicular to the film plane. The values of the exchange length $l$, the static field $H$, the Landau-Lifshitz damping $g$, and the driving frequency $\omega$ are $0.05 d, 1.1 \cdot 4 \pi M_{0}, 0.01$, and $0.42 \cdot 4 \pi \gamma M_{0}$, respectively, where $M_{0}$ is the magnetization and $\gamma$ the gyromagnetic ratio. The solid and dashed lines denote the analytically found neutral curves $h_{S}$ and $h_{H}$, respectively. The squares and triangles are numerical results based on a Galerkin expansion 13. 
Fig. 2 --- F.J. Elmer

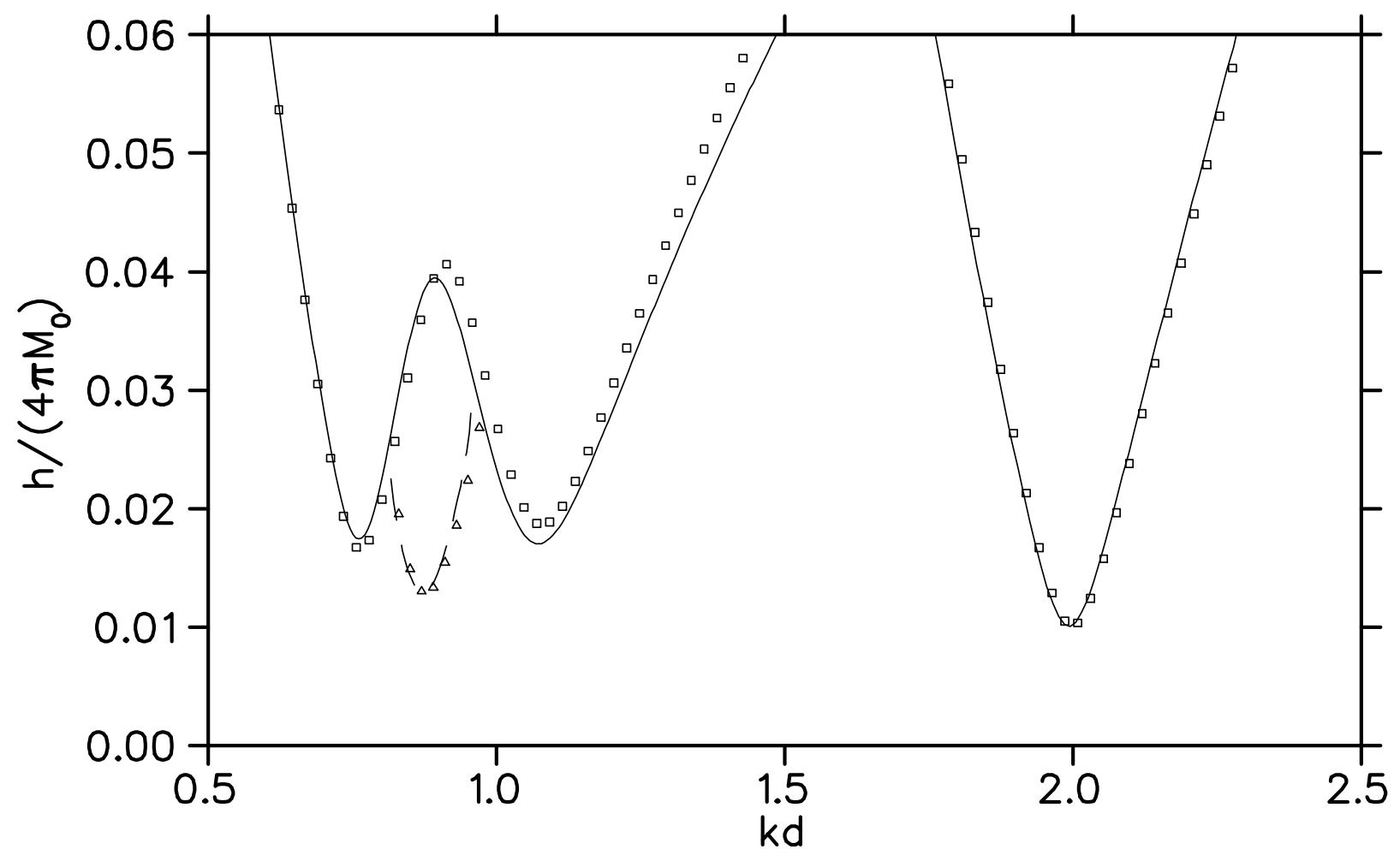


Fig. 1(a) --- F.J. Elmer

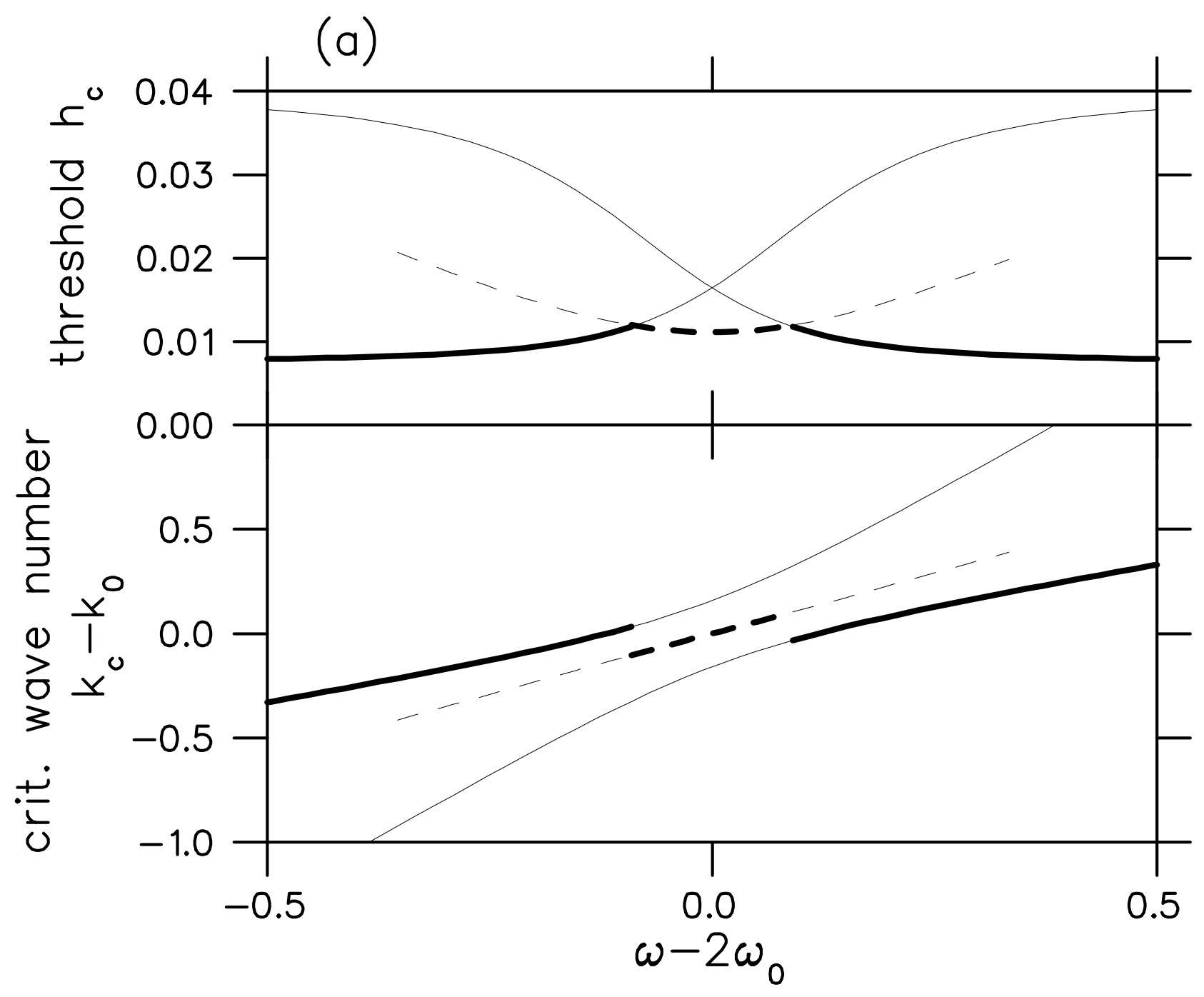


Fig. 1(b) --- F.J. Elmer

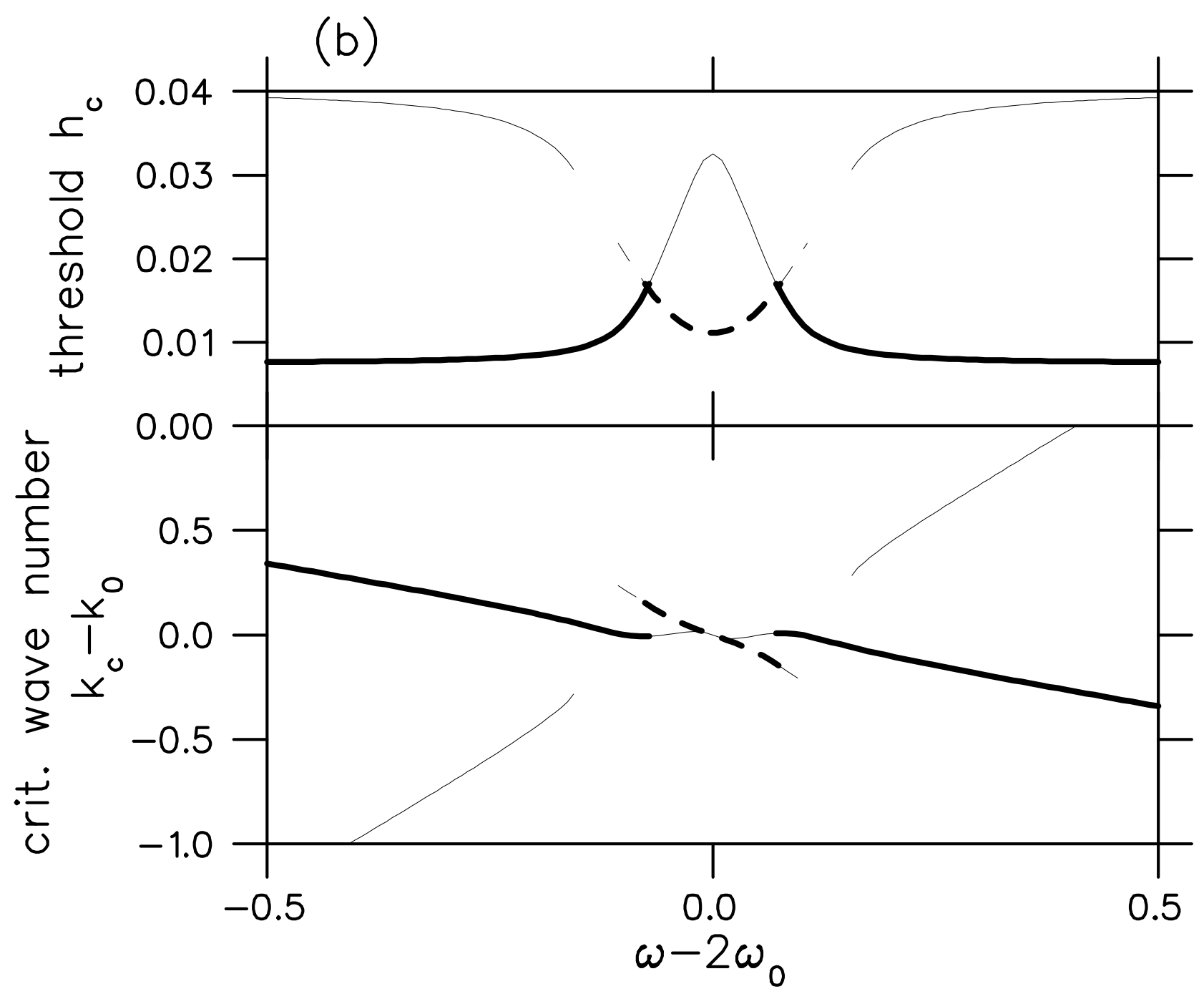

